\title{
A hybrid Caputo fractional modeling for thermostat with hybrid boundary value conditions
}

\author{
Dumitru Baleanu ${ }^{1,2,4}$, Sina Etemad ${ }^{3}$ and Shahram Rezapour ${ }^{3,4^{*}}$
}

"Correspondence:
rezapourshahram@yahoo.ca;
sh.rezapour@azaruniv.ac.ir
${ }^{3}$ Department of Mathematics,
Azarbaijan Shahid Madani
University, Tabriz, Iran
${ }^{4}$ Department of Medical Research,
China Medical University Hospital,
China Medical University, Taichung,
Taiwan
Full list of author information is
available at the end of the article

available at the end of the article

\begin{abstract}
We provide an extension for the second-order differential equation of a thermostat model to the fractional hybrid equation and inclusion versions. We consider boundary value conditions of this problem in the form of the hybrid conditions. To prove the existence of solutions for our hybrid fractional thermostat equation and inclusion versions, we apply the well-known Dhage fixed point theorems for single-valued and set-valued maps. Finally, we give two examples to illustrate our main results.
\end{abstract}

MSC: Primary 34A08; secondary 34A12

Keywords: Caputo fractional derivative; Hybrid fractional differential equation and inclusion; Thermostat modeling

\section{Introduction}

Maybe it would have been a funny thing to talk about the possibility of mathematics helping the environment a few years ago. In fact, mathematics can play a role if modeling comes to the point where we can do some of the chemical testing on computers. Thus, it is very important we increase our abilities in modern modeling by working on complicated fractional integro-differential equations and inclusions. As is well known, there have been studied different types of hybrid equations by many researchers (see, for example, [1-4] and [5]). One of the significant strategies is reviewing of hybrid models of different phenomena.

In 2010, Dhage and Lakshmikantham introduced hybrid differential equations [6]. In 2011, Zhao et al. extended Dhage's work to fractional order and investigated the hybrid fractional differential equations [4]. In 2012, Sun et al. studied the fractional hybrid twopoint boundary value problem

$$
\left\{\begin{array}{l}
\mathcal{D}_{0}^{p}\left[\frac{z(t)}{h(t, z(t))}\right]+g(t, z(t))=0 \quad(t \in[0,1], p \in(1,2]), \\
z(0)=z(1)=0
\end{array}\right.
$$

By applying a Dhage's fixed point theorem in Banach algebra under the mixed Carathéodory and Lipschitz conditions, they obtained some existence results. In 2015,

(c) The Author(s) 2020. This article is licensed under a Creative Commons Attribution 4.0 International License, which permits use, sharing, adaptation, distribution and reproduction in any medium or format, as long as you give appropriate credit to the original author(s) and the source, provide a link to the Creative Commons licence, and indicate if changes were made. The images or other third party material in this article are included in the article's Creative Commons licence, unless indicated otherwise in a credit line to the material. If material is not included in the article's Creative Commons licence and your intended use is not permitted by statutory regulation or exceeds the permitted use, you will need to obtain permission directly from the copyright holder. To view a copy of this licence, visit http://creativecommons.org/licenses/by/4.0/. 
Hilal and Kajouni discussed the existence of extremal solutions for the Caputo hybrid boundary value problem

$$
\left\{\begin{array}{l}
{ }^{c} \mathcal{D}_{0}^{p}\left[\frac{z(t)}{h(t, z(t))}\right]=g(t, z(t))=0 \quad(t \in J=[0, T]), \\
a \frac{z(0)}{h(0, z(0))}+b \frac{z(T)}{h(T, z(T))}=c,
\end{array}\right.
$$

where $p \in(0,1), a, b$ and $c$ are some real constants with $a+b \neq 0$ and the functions $h$ : $J \times \mathbb{R} \rightarrow \mathbb{R} \backslash\{0\}$ and $g: J \times \mathbb{R} \rightarrow \mathbb{R}$ are continuous [3]. Some months later, Baleanu et al. reviewed some existence results for the Caputo fractional hybrid inclusion problem

$$
{ }^{c} \mathcal{D}_{0}^{p}\left(\frac{z(t)}{h\left(t, z(t), \mathcal{I}_{0}^{\alpha_{1}} z(t), \ldots, \mathcal{I}_{0}^{\alpha_{n}} z(t)\right)}\right) \in \mathcal{H}\left(t, z(t), \mathcal{I}_{0}^{\beta_{1}} z(t), \ldots, \mathcal{I}_{0}^{\beta_{k}} z(t)\right),
$$

for all $t \in[0,1]$, with boundary value conditions $z(0)=z_{0}^{*}$ and $z(1)=z_{1}^{*}$, where $p \in(1,2]$, ${ }^{c} \mathcal{D}_{0}^{p}$ and $\mathcal{I}_{0}^{\gamma}$ denote the Caputo derivative operator of the fractional order $p$ and the Riemann-Liouville integral operator of the fractional order $\gamma \in\left\{\alpha_{i}, \beta_{j}\right\} \subset(0, \infty)$ for $i=$ $1, \ldots n$ and $j=1, \ldots, k$, respectively. They used the Dhage fixed point theorem for multivalued mappings for the existence result [5].

In 2006, Infante and Webb formulated a model of thermostat insulated at $t=0$ with the controller at $t=1$ :

$$
\left\{\begin{array}{l}
z^{\prime \prime}(t)+\Phi(t, z(t))=0 \quad(t \in[0,1]) \\
z^{\prime}(0)=0, \quad \lambda z^{\prime}(1)+z(\eta)=0
\end{array}\right.
$$

where $\eta \in[0,1]$ is a real constant and $\lambda>0$ is a parameter [7]. According to this secondorder model, the thermostat adds or discharges heat depending on the temperature detected by the sensor at $t=\eta[7])$. They obtained existence results for the boundary value problem by applying the fixed point index theory on Hammerstein integral equations [7]. Some years later, Nieto and Pimentel studied the fractional-order version of the problem

$$
\left\{\begin{array}{l}
{ }^{c} \mathcal{D}^{p} z(t)+\Phi(t, z(t))=0 \quad(t \in[0,1]), \\
z^{\prime}(0)=0, \quad \lambda^{c} \mathcal{D}^{p-1} z(1)+z(\eta)=0,
\end{array}\right.
$$

where ${ }^{c} \mathcal{D}^{p}$ denotes the fractional derivative of Caputo type of order $p \in(1,2]$ and $\lambda>0$ and $\eta \in[0,1]$ are given real constants [8].

It is well known that a thermostat is a component which senses the temperature of a physical system and performs actions so that the system's temperature is maintained near a desired set-point. Many researchers have reviewed different models for thermostat systems. They have been provided some models for thermostat systems (see, for example, [9-11]).

By using the main idea of $[3,5,7]$ and $[8]$ and mixing those with the thermostat models, we are going to investigate the fractional hybrid problem for the thermostat model

$$
{ }^{c} \mathcal{D}_{0}^{p}\left(\frac{z(t)}{h(t, z(t))}\right)+\Phi(t, z(t))=0 \quad(t \in[0,1])
$$


with the hybrid boundary value conditions

$$
\left\{\begin{array}{l}
\left.\mathcal{D}\left(\frac{z(t)}{h(t, z(t))}\right)\right|_{t=0}=0 \\
\left.\lambda^{c} \mathcal{D}_{0}^{p-1}\left(\frac{z(t)}{h(t, z(t))}\right)\right|_{t=1}+\left.\left(\frac{z(t)}{h(t, z(t))}\right)\right|_{t=\eta}=0
\end{array}\right.
$$

where $p \in(1,2], p-1 \in(0,1], \eta \in[0,1], \mathcal{D}=\frac{\mathrm{d}}{\mathrm{d} t}, \lambda$ is a positive real parameter, ${ }^{c} \mathcal{D}_{0}^{\gamma}$ is the Caputo derivation of fractional order $\gamma \in\{p, p-1\}$, the function $\Phi:[0,1] \times \mathbb{R} \rightarrow \mathbb{R}$ is continuous and $h \in C([0,1] \times \mathbb{R}, \mathbb{R} \backslash\{0\})$.

Also, we investigate the hybrid fractional differential inclusion model of the thermostat as

$$
-{ }^{c} \mathcal{D}_{0}^{p}\left(\frac{z(t)}{h(t, z(t))}\right) \in \mathcal{G}(t, z(t)), \quad t \in[0,1]
$$

with the three-point hybrid boundary value conditions

$$
\left\{\begin{array}{l}
\left.\mathcal{D}\left(\frac{z(t)}{h(t, z(t))}\right)\right|_{t=0}=0 \\
\left.\lambda^{c} \mathcal{D}_{0}^{p-1}\left(\frac{z(t)}{h(t, z(t))}\right)\right|_{t=1}+\left.\left(\frac{z(t)}{h(t, z(t))}\right)\right|_{t=\eta}=0
\end{array}\right.
$$

where $\mathcal{G}:[0,1] \times \mathbb{R} \rightarrow \mathcal{P}(\mathbb{R})$ is a multi-valued map.

Let $p>0$ be a real number with $n-1<p<n$ and the function $z:[a, b] \rightarrow \mathbb{R}$ integrable (or $z \in C([a, b], \mathbb{R}))$. The Riemann-Liouville fractional integral of the function $z$ is defined by

$$
\mathcal{J}_{0}^{p} z(t)=\frac{1}{\Gamma(p)} \int_{0}^{t}(t-\tau)^{p-1} z(\tau) \mathrm{d} \tau \quad(t>0)
$$

whenever the integral exists $[12,13]$. The Caputo fractional derivative of a function $z \in$ $C^{(n)}([a, b], \mathbb{R})$ is defined by

$$
{ }^{c} \mathcal{D}_{0}^{p} z(t)=\frac{1}{\Gamma(n-p)} \int_{0}^{t}(t-\tau)^{n-p-1} z^{(n)}(\tau) \mathrm{d} \tau,
$$

where $n-1<p<n$ with $n=[p]+1[12,13]$. It is well known that a general solution for the fractional differential equation ${ }^{c} \mathcal{D}_{0}^{p} z(t)=0$ is given by $z(t)=b_{0}+b_{1} t+b_{2} t^{2}+\cdots+b_{n-1} t^{n-1}$, where $b_{0}, \ldots, b_{n-1}$ are some real numbers and $n=[p]+1$ [14]. Also, for every positive real number $T$ and every continuous function $z$ on the interval $[0, T]$, the relation

$$
\left(\mathcal{I}_{0}^{p_{C}} \mathcal{D}_{0}^{p}\right) z(t)=z(t)+\sum_{j=0}^{n-1} b_{j} t^{j}=z(t)+b_{0}+b_{1} t+b_{2} t^{2}+\cdots+b_{n-1} t^{n-1}
$$

holds, where $b_{0}, \ldots, b_{n-1}$ are some real constants and $n=[p]+1[14]$.

Assume that $\left(\mathcal{M},\|\cdot\|_{\mathcal{M}}\right)$ is a normed space. We denote by $\mathcal{P}(\mathcal{M}), \mathcal{P}_{c l}(\mathcal{M}), \mathcal{P}_{b}(\mathcal{M})$, $\mathcal{P}_{c p}(\mathcal{M})$ and $\mathcal{P}_{c v}(\mathcal{M})$ the set of all subsets of $\mathcal{M}$, the set of all closed subsets of $\mathcal{M}$, the set of all bounded subsets of $\mathcal{M}$, the set of all compact subsets of $\mathcal{M}$, and the set of all convex subsets of $\mathcal{M}$, respectively. A set-valued map $\mathcal{G}$ has convex values if the set $\mathcal{G}(z)$ is convex for each element $z \in \mathcal{M}$. Moreover, we say that the set-valued map $\mathcal{G}$ is an upper 
semi-continuous (u.s.c.) whenever for each $z^{*} \in \mathcal{M}$, the set $\mathcal{G}\left(z^{*}\right)$ belongs to $\mathcal{P}_{c l}(\mathcal{M})$ and for every open set $\mathcal{V}$ containing $\mathcal{G}\left(z^{*}\right)$, there exists an open neighborhood $\mathcal{U}_{0}^{*}$ of $z^{*}$ such that $\mathcal{G}\left(\mathcal{U}_{0}^{*}\right) \subseteq \mathcal{V}$ [15]. An element $z^{*} \in \mathcal{M}$ is called a fixed point for the multi-valued map $\mathcal{G}: \mathcal{M} \rightarrow \mathcal{P}(\mathcal{M})$ whenever $z^{*} \in \mathcal{G}\left(z^{*}\right)$ [15]. The set of all fixed points of the multifunction $\mathcal{G}$ is denoted by $\operatorname{Fix}(\mathcal{G})$ [15].

Let $\left(\mathcal{M}, d_{\mathcal{M}}\right)$ be a metric space. For each two subsets $A_{1}, A_{2} \in \mathcal{P}(\mathcal{M})$, the PompeiuHausdorff metric $\mathrm{PH}_{d}: \mathcal{P}(\mathcal{M}) \times \mathcal{P}(\mathcal{M}) \rightarrow \mathbb{R} \cup\{\infty\}$ is defined by

$$
\operatorname{PH}_{d_{\mathcal{M}}}\left(A_{1}, A_{2}\right)=\max \left\{\sup _{a_{1} \in A_{1}} d_{\mathcal{M}}\left(a_{1}, A_{2}\right), \sup _{a_{2} \in A_{2}} d_{\mathcal{M}}\left(A_{1}, a_{2}\right)\right\}
$$

where $d_{\mathcal{M}}\left(A_{1}, a_{2}\right)=\inf _{a_{1} \in A_{1}} d_{\mathcal{M}}\left(a_{1}, a_{2}\right)$ and $d_{\mathcal{M}}\left(a_{1}, A_{2}\right)=\inf _{a_{2} \in A_{2}} d_{\mathcal{M}}\left(a_{1}, a_{2}\right)$ [15]. A multivalued function $\mathcal{G}: \mathcal{M} \rightarrow \mathcal{P}_{c l}(\mathcal{M})$ is said to be Lipschitzian with Lipschitz constant $k>0$ whenever $\mathrm{PH}_{d_{\mathcal{M}}}\left(\mathcal{G}\left(z_{1}\right), \mathcal{G}\left(z_{2}\right)\right) \leq k d_{\mathcal{M}}\left(z_{1}, z_{2}\right)$ holds for each $z_{1}, z_{2} \in \mathcal{M}$. A Lipschitz map $\mathcal{G}$ is called contraction whenever $k \in(0,1)$ [15]. A set-valued operator $\mathcal{G}:[0,1] \rightarrow \mathcal{P}_{c l}(\mathbb{R})$ is called measurable whenever the function $t \longmapsto d_{\mathcal{M}}(\omega, \mathcal{G}(t))=\inf \{|\omega-\nu|: v \in \mathcal{G}(t)\}$ is measurable for all real constant $\omega[15,16]$. The graph of a set-valued function $\mathcal{G}: \mathcal{M} \rightarrow \mathcal{P}_{c l}(\mathcal{Q})$ is defined by $\operatorname{Graph}(\mathcal{G})=\left\{\left(z_{1}, z_{2}\right) \in \mathcal{M} \times \mathcal{Q}: w^{*} \in \mathcal{G}(z)\right\}$ [15]. We say that graph of $\mathcal{G}$ is closed whenever for each sequence $\left\{z_{n}\right\}_{n \geq 1}$ in $\mathcal{M}$ and $\left\{w_{n}\right\}_{n \geq 1}$ in $\mathcal{Q}, z_{n} \rightarrow z_{0}, w_{n} \rightarrow w_{0}$ and $w_{n} \in \mathcal{G}\left(z_{n}\right)$, wh have $w_{0} \in \mathcal{G}\left(z_{0}\right)[16]$.

A multifunction $\mathcal{G}$ is said to be completely continuous operator whenever the set $\mathcal{G}(\mathcal{W})$ is relatively compact for all $\mathcal{W} \in \mathcal{P}_{b}(\mathcal{M})$. If the multifunction $\mathcal{G}: \mathcal{M} \rightarrow \mathcal{P}_{c l}(\mathcal{Q})$ is an upper semi-continuous, then $\operatorname{Graph}(\mathcal{G})$ is a subset of the product space $\mathcal{M} \times \mathcal{Q}$ with closeness property. Conversely, if the set-valued mapping $\mathcal{G}$ is completely continuous and has a closed graph, then $\mathcal{G}$ is an upper semi-continuous ([15], Proposition 2.1). A set-valued map $\mathcal{G}:[0,1] \times \mathbb{R} \rightarrow \mathcal{P}(\mathbb{R})$ is said to be Carathéodory multifunction whenever $t \mapsto \mathcal{G}(t, z)$ is measurable mapping for all $z \in \mathbb{R}$ and $z \mapsto \mathcal{G}(t, z)$ is upper semi-continuous mapping for almost all $t \in[0,1][15,16]$. Also, a Carathéodory multifunction $\mathcal{G}:[0,1] \times \mathbb{R} \rightarrow \mathcal{P}(\mathbb{R})$ is said to be $\mathcal{L}^{1}$-Carathéodory whenever for each constant $\mu>0$ there exists a function $\phi_{\mu} \in \mathcal{L}^{1}\left([0,1], \mathbb{R}^{+}\right)$such that

$$
\|\mathcal{G}(t, z)\|=\sup _{t \in[0,1]}\{|s|: s \in \mathcal{G}(t, z)\} \leq \phi_{\mu}(t)
$$

for all $|z| \leq \mu$ and for almost all $t \in[0,1][15,16]$. The set of selections of a multifunction $\mathcal{G}$ at point $z \in C([0,1], \mathbb{R})$ is defined by

$$
(S E L)_{\mathcal{G}, z}:=\left\{v \in \mathcal{L}^{1}([0,1], \mathbb{R}): v(t) \in \mathcal{G}(t, z(t))\right\}
$$

for almost all $t \in[0,1][15,16]$. Let $\mathcal{G}$ be a set-valued map. It is known that $(S E L)_{\mathcal{G}, z} \neq \emptyset$ for all $z \in C([0,1], \mathcal{M})$ whenever $\operatorname{dim} \mathcal{M}<\infty[15]$. We need the following results.

Lemma 1 (Dhage's nonlinear alternative of Schaefer's type [17]) Let $\mathcal{M}$ be a Banach algebra. For each positive number $\varepsilon \in \mathbb{R}$, consider the open ball $V_{\varepsilon}(0)$ and the closed ball $\bar{V}_{\varepsilon}(0)$. Suppose that two operators $\mathcal{B}_{1}: \mathcal{M} \rightarrow \mathcal{M}$ and $\mathcal{B}_{2}: \bar{V}_{\varepsilon}(0) \rightarrow \mathcal{M}$ satisfy the following conditions:

(i) $\mathcal{B}_{1}$ is an operator including Lipschitzian property with a Lipschitz constant $l^{*}$;

(ii) $\mathcal{B}_{2}$ has complete continuity property; 
(iii) $l^{*} M_{0}^{*}<1$, so that $M_{0}^{*}=\left\|\mathcal{B}_{2}\left(\bar{V}_{\varepsilon}(0)\right)\right\|_{\mathcal{M}}=\sup \left\{\left\|\mathcal{B}_{2} z\right\|_{\mathcal{M}}: z \in \bar{V}_{\varepsilon}(0)\right\}$.

Then either

(a) The operator equation $\mathcal{B}_{1} z \mathcal{B}_{2} z=z$ has a solution in $\bar{V}_{\varepsilon}(0)$, or

(b) there is an element $v^{*} \in \mathcal{M}$ with $\left\|v^{*}\right\|_{\mathcal{M}}=\varepsilon$ such that $\mu \mathcal{B}_{1} v^{*} \mathcal{B}_{2} v^{*}=v^{*}$ for some $\mu \in(0,1)$.

Lemma $2([18])$ Suppose that $\mathcal{M}$ is a separable Banach space, $\mathcal{G}:[0,1] \times \mathcal{M} \rightarrow \mathcal{P}_{c p, c v}(\mathcal{M})$ is an $\mathcal{L}^{1}$-Carathéodory multifunction and $\Upsilon: \mathcal{L}^{1}([0,1], \mathcal{M}) \rightarrow C([0,1], \mathcal{M})$ is a linear continuous mapping. Then

$$
\Upsilon \circ(S E L)_{\mathcal{G}}: C([0,1], \mathcal{M}) \rightarrow \mathcal{P}_{c p, c v}(C([0,1], \mathcal{M}))
$$

is an operator in the product space $C([0,1], \mathcal{M}) \times C([0,1], \mathcal{M})$ with action $z \mapsto(\Upsilon \circ$ $\left.(S E L)_{\mathcal{G}}\right)(z)=\Upsilon\left((S E L)_{\mathcal{G}, z}\right)$ having closed graph property.

Lemma 3 ([19]) Let $M$ be a Banach algebra. Suppose that there exist a single-valued map $\mathcal{B}_{1}: \mathcal{M} \rightarrow \mathcal{M}$ and a multi-valued map $\mathcal{B}_{2}: \mathcal{M} \rightarrow \mathcal{P}_{c p, c v}(\mathcal{M})$ such that

(i) $\mathcal{B}_{1}$ is an operator including Lipschitzian property with a Lipschitz constant $l^{*}$;

(ii) $\mathcal{B}_{2}$ is an operator including upper semi-continuity and compactness property;

(iii) $2 l^{*} M_{0}^{*}<1$ so that $M_{0}^{*}=\left\|\mathcal{B}_{2}(\mathcal{M})\right\|$.

Then either

(a) there is a solution in $\mathcal{M}$ for the operator inclusion $z \in \mathcal{B}_{1} z \mathcal{B}_{2} z$, or

(b) The set $\Sigma^{*}=\left\{v^{*} \in \mathcal{M} \mid \mu v^{*} \in \mathcal{B}_{1} v^{*} \mathcal{B}_{2} v^{*}, \mu>1\right\}$ is not bounded.

\section{Main results}

Consider the Banach space $\mathcal{M}=C_{\mathbb{R}}([0,1])$ with the norm

$$
\|z\|_{\mathcal{M}}=\sup \{|z(t)|: t \in[0,1]\} .
$$

Now, we provide our first key result.

Lemma 4 Let $\rho \in \mathcal{M}$. A function $z_{0}$ is a solution for the hybrid fractional equation

$$
{ }^{c} \mathcal{D}_{0}^{p}\left(\frac{z(t)}{h(t, z(t))}\right)+\rho(t)=0, \quad t \in[0,1], p \in(1,2],
$$

with the three-point hybrid boundary conditions

$$
\begin{aligned}
& \left.\mathcal{D}\left(\frac{z(t)}{h(t, z(t))}\right)\right|_{t=0}=0, \\
& \left.\lambda^{c} \mathcal{D}_{0}^{p-1}\left(\frac{z(t)}{h(t, z(t))}\right)\right|_{t=1}+\left.\left(\frac{z(t)}{h(t, z(t))}\right)\right|_{t=\eta}=0,
\end{aligned}
$$

if and only if $z_{0}$ is a solution for the integral equation

$$
\begin{aligned}
z(t)= & h(t, z(t))\left[-\int_{0}^{t} \frac{(t-\tau)^{p-1}}{\Gamma(p)} \rho(\tau) \mathrm{d} \tau\right. \\
& \left.+\int_{0}^{\eta} \frac{(\eta-\tau)^{p-1}}{\Gamma(p)} \rho(\tau) \mathrm{d} \tau+\lambda \int_{0}^{1} \rho(\tau) \mathrm{d} \tau\right] .
\end{aligned}
$$


Proof First, assume that $z_{0}$ is a solution for the hybrid fractional equation (5). Then there exist constants $b_{0}, b_{1} \in \mathbb{R}$ such that

$$
z_{0}(t)=h\left(t, z_{0}(t)\right)\left[-\int_{0}^{t} \frac{(t-\tau)^{p-1}}{\Gamma(p)} \rho(\tau) \mathrm{d} \tau+b_{0}+b_{1} t\right] .
$$

Then

$$
\mathcal{D}\left(\frac{z_{0}(t)}{h\left(t, z_{0}(t)\right)}\right)=-\int_{0}^{t} \frac{(t-\tau)^{p-2}}{\Gamma(p-1)} \rho(\tau) \mathrm{d} \tau+b_{1}
$$

and

$$
{ }^{c} \mathcal{D}_{0}^{p-1}\left(\frac{z_{0}(t)}{h\left(t, z_{0}(t)\right)}\right)=-\int_{0}^{t} \rho(\tau) \mathrm{d} \tau+b_{1} \frac{t^{2-p}}{\Gamma(3-p)} .
$$

Thus, $b_{1}=0$ and

$$
b_{0}=\int_{0}^{1} \rho(\tau) \mathrm{d} \tau+\int_{0}^{\eta} \frac{(\eta-\tau)^{p-1}}{\Gamma(p)} \rho(\tau) \mathrm{d} \tau .
$$

By substituting the values $b_{0}$ and $b_{1}$ in (8), we get

$$
z_{0}(t)=h\left(t, z_{0}(t)\right)\left[-\int_{0}^{t} \frac{(t-\tau)^{p-1}}{\Gamma(p)} \rho(\tau) \mathrm{d} \tau+\int_{0}^{\eta} \frac{(\eta-\tau)^{p-1}}{\Gamma(p)} \rho(\tau) \mathrm{d} \tau+\lambda \int_{0}^{1} \rho(\tau) \mathrm{d} \tau\right] .
$$

This means that $z_{0}$ is a solution function for the fractional integral equation (7). Conversely, it can easily be seen that $z_{0}$ is a solution function for the fractional hybrid problem (5)-(6) whenever $z_{0}$ is a solution function for the fractional integral equation (7).

Theorem 5 Assume that $h \in C([0,1] \times \mathbb{R}, \mathbb{R} \backslash\{0\})$ and $\Phi \in C([0,1] \times \mathbb{R}, \mathbb{R})$ and

$(\mathcal{A} 1)$ there is a bounded mapping $\kappa:[0,1] \rightarrow \mathbb{R}^{+}$such that

$$
\left|h\left(t, z_{1}\right)-h\left(t, z_{2}\right)\right| \leq \kappa(t)\left|z_{1}(t)-z_{2}(t)\right|
$$

for all $z_{1}, z_{2} \in \mathbb{R}$ and $t \in[0,1]$,

$(\mathcal{A} 2)$ there are a continuous non-decreasing map $\chi:[0, \infty) \rightarrow(0, \infty)$ and a continuous function $g:[0,1] \rightarrow \mathbb{R}^{+}$such that

$$
|\Phi(t, z)| \leq g(t) \chi(\|z\|)
$$

for $t \in[0,1]$ and for all $z \in \mathbb{R}$,

$(\mathcal{A} 3)$ there is a positive real number $\varepsilon$ such that

$$
\varepsilon>\frac{H^{*} \Delta^{*} G^{*} \chi(\|z\|)}{1-\kappa^{*} \Delta^{*} G^{*} \chi(\|z\|)},
$$

where $H^{*}=\sup _{t \in[0,1]}|h(t, 0)|, G^{*}=\sup _{t \in[0,1]}|g(t)|, \kappa^{*}=\sup _{t \in[0,1]}|\kappa(t)|$ and

$$
\Delta^{*}=\frac{1}{\Gamma(p+1)}+\frac{\eta^{p}}{\Gamma(p+1)}+\lambda
$$


If $\kappa^{*} \Delta^{*} G^{*} \chi(\|z\|)<1$, then the hybrid fractional problem (1)-(2) has at least one solution.

Proof Consider the closed ball $\bar{V}_{\varepsilon}(0):=\left\{z \in \mathcal{M}:\|z\|_{\mathcal{M}} \leq \varepsilon\right\}$ in the Banach space $\mathcal{M}$, where $\varepsilon$ satisfies the inequality (9). By using Lemma 4 and the fractional integral equation (7), we define two operators $\mathcal{B}_{1}, \mathcal{B}_{2}: \bar{V}_{\epsilon}(0) \rightarrow \mathcal{M}$ by $\left(\mathcal{B}_{1} z\right)(t)=h(t, z(t))$ and

$$
\begin{aligned}
\left(\mathcal{B}_{2} z\right)(\tau)= & -\int_{0}^{t} \frac{(t-\tau)^{p-1}}{\Gamma(p)} \Phi(\tau, z(\tau)) \mathrm{d} \tau+\lambda \int_{0}^{1} \Phi(\tau, z(\tau)) \mathrm{d} \tau \\
& +\int_{0}^{\eta} \frac{(\eta-\tau)^{p-1}}{\Gamma(p)} \Phi(\tau, z(\tau)) \mathrm{d} \tau .
\end{aligned}
$$

It is clear that $z \in \mathcal{M}$ as a solution for the fractional hybrid problem (1)-(2) satisfies the operator equation $\mathcal{B}_{1} z \mathcal{B}_{2} z=z$. To prove this subject, by applying assumptions of Lemma 1 , we show that there exists such a solution. In first step, it is proved that the operator $\mathcal{B}_{1}$ is Lipschitzian operator on normed space $\mathcal{M}$ with Lipschitz constant $\kappa^{*}=\sup _{t \in[0,1]}|\kappa(t)|$. Let $z_{1}, z_{2} \in \mathcal{M}$. By using the assumption $(\mathcal{A} 1)$, we have

$$
\begin{aligned}
\left|\left(\mathcal{B}_{1} z_{1}\right)(t)-\left(\mathcal{B}_{1} z_{2}\right)(t)\right| & =\left|h\left(t, z_{1}(t)\right)-h\left(t, z_{2}(t)\right)\right| \\
& \leq \kappa(t)\left|z_{1}(t)-z_{2}(t)\right|
\end{aligned}
$$

for each $z_{1}, z_{2} \in \bar{V}_{\varepsilon}(0)$. By taking the supremum over $[0,1]$, one can deduce that

$$
\left\|\mathcal{B}_{1} z_{1}-\mathcal{B}_{1} z_{2}\right\|_{\mathcal{M}} \leq \kappa^{*}\left\|z_{1}-z_{2}\right\|_{\mathcal{M}}
$$

for all $z_{1}, z_{2} \in \bar{V}_{\varepsilon}(0)$. This shows that $\mathcal{B}_{1}$ is Lipschitzian operator on $\bar{V}_{\varepsilon}(0)$ with Lipschitz constant $\kappa^{*}$. To establish the complete continuity of the operator $\mathcal{B}_{2}$ on $\bar{V}_{\varepsilon}(0)$, we first prove that the operator $\mathcal{B}_{2}$ is continuous on $\bar{V}_{\varepsilon}(0)$. To this aim, suppose that $\left\{z_{n}\right\}$ is a sequence in the open ball $\bar{V}_{\varepsilon}(0)$ with $z_{n} \rightarrow z$, where $z$ is an element of $\bar{V}_{\varepsilon}(0)$. Since $\Phi$ is continuous on $[0,1] \times \mathbb{R}, \lim _{n \rightarrow \infty} \Phi\left(t, z_{n}(t)\right)=\Phi(t, z(t))$. By using the Lebesgue dominated convergence theorem, we conclude that

$$
\begin{aligned}
\lim _{n \rightarrow \infty}\left(\mathcal{B}_{2} z_{n}\right)(t)= & -\int_{0}^{t} \frac{(t-\tau)^{p-1}}{\Gamma(p)} \lim _{n \rightarrow \infty} \Phi\left(\tau, z_{n}(\tau)\right) \mathrm{d} \tau \\
& +\lambda \int_{0}^{1} \lim _{n \rightarrow \infty} \Phi\left(\tau, z_{n}(\tau)\right) \mathrm{d} \tau \\
& +\int_{0}^{\eta} \frac{(\eta-\tau)^{p-1}}{\Gamma(p)} \lim _{n \rightarrow \infty} \Phi\left(\tau, z_{n}(\tau)\right) \mathrm{d} \tau \\
= & -\int_{0}^{t} \frac{(t-\tau)^{p-1}}{\Gamma(p)} \Phi(\tau, z(\tau)) \mathrm{d} \tau+\lambda \int_{0}^{1} \Phi(\tau, z(\tau)) \mathrm{d} \tau \\
& +\int_{0}^{\eta} \frac{(\eta-\tau)^{p-1}}{\Gamma(p)} \Phi(\tau, z(\tau)) \mathrm{d} \tau \\
= & \left(\mathcal{B}_{2} z\right)(t)
\end{aligned}
$$

for all $t \in[0,1]$. Thus, $\mathcal{B}_{2} z_{n} \rightarrow \mathcal{B}_{2} z$ and so $\mathcal{B}_{2}$ is a continuous operator on $\bar{V}_{\varepsilon}(0)$. 
In the next step, the uniform boundedness of operator $\mathcal{B}_{2}$ on $\bar{V}_{\varepsilon}(0)$ is checked. By assumption $(\mathcal{A} 2)$, we have

$$
\begin{aligned}
\left|\left(\mathcal{B}_{2} z\right)(t)\right|= & \int_{0}^{t} \frac{(t-\tau)^{p-1}}{\Gamma(p)}|\Phi(\tau, z(\tau))| \mathrm{d} \tau+\lambda \int_{0}^{1}|\Phi(\tau, z(\tau))| \mathrm{d} \tau \\
& +\int_{0}^{\eta} \frac{(\eta-\tau)^{p-1}}{\Gamma(p)}|\Phi(\tau, z(\tau))| \mathrm{d} \tau \\
\leq & \frac{t^{p}}{\Gamma(p+1)} g(\tau) \chi(\|z\|)+\lambda g(\tau) \chi(\|z\|) \\
& +\frac{\eta^{p}}{\Gamma(p+1)} g(\tau) \chi(\|z\|)
\end{aligned}
$$

for all $t \in[0,1]$ and $z \in \bar{V}_{\varepsilon}(0)$. Thus $\left\|\mathcal{B}_{2} z\right\| \leq G^{*} \chi(\|z\|) \Delta^{*}$, where $\Delta^{*}$ is given in (10). This implies that the set $\mathcal{B}_{2}\left(\bar{V}_{\varepsilon}(0)\right)$ is an uniformly bounded in the normed space $\mathcal{M}$. Now, the equi-continuity of the operator $\mathcal{B}_{2}$ is investigated. To this aim, assume that $t_{1}, t_{2} \in[0,1]$ with $t_{1}<t_{2}$. Then we have

$$
\begin{aligned}
\left|\left(\mathcal{B}_{2} z\right)\left(t_{2}\right)-\left(\mathcal{B}_{2} z\right)\left(t_{1}\right)\right|= & \mid \int_{0}^{t_{2}} \frac{\left(t_{2}-\tau\right)^{p-1}}{\Gamma(p)} \Phi(\tau, z(\tau)) \mathrm{d} \tau \\
& -\int_{0}^{t_{1}} \frac{\left(t_{1}-\tau\right)^{p-1}}{\Gamma(p)} \Phi(\tau, z(\tau)) \mathrm{d} \tau \mid \\
\leq & G^{*} \chi(\|z\|)\left[\int_{0}^{t_{1}}\left(\frac{\left(t_{2}-\tau\right)^{p-1}}{\Gamma(p)}-\frac{\left(t_{1}-\tau\right)^{p-1}}{\Gamma(p)}\right) \mathrm{d} \tau\right. \\
& \left.+\int_{t_{1}}^{t_{2}} \frac{\left(t_{2}-\tau\right)^{p-1}}{\Gamma(p)} \mathrm{d} \tau\right] .
\end{aligned}
$$

It is observed that the right-hand side of the inequalities converges to zero independently of $z \in \bar{V}_{\varepsilon}(0)$ as $t_{1} \rightarrow t_{2}$. Therefore the operator $\mathcal{B}_{2}$ is equi-continuous. Now by using the Arzela-Ascoli theorem, we conclude that the operator $\mathcal{B}_{2}$ is complete continuous on $\bar{V}_{\varepsilon}(0)$.

On the other hand by using $(\mathcal{A} 3)$, we have

$$
\begin{aligned}
M_{0}^{*} & =\left\|\mathcal{B}_{2}\left(\bar{V}_{\varepsilon}(0)\right)\right\|_{\mathcal{M}}=\sup \left\{\left|\mathcal{B}_{2} z\right|: z \in \bar{V}_{\varepsilon}(0)\right\} \\
& =G^{*} \chi(\|z\|)\left[\frac{1}{\Gamma(p+1)}+\frac{\eta^{p}}{\Gamma(p+1)}+\lambda\right] \\
& =G^{*} \chi(\|z\|) \Delta^{*} .
\end{aligned}
$$

By putting $l^{*}=\kappa^{*}$, we get $G^{*} l^{*}<1$ and so the assumptions of Lemma 1 hold and so one of the conditions (a) or (b) in Lemma 1 holds. For some $\mu \in(0,1)$, assume that $z$ satisfies the operator equation $z=\mu \mathcal{B}_{1} z \mathcal{B}_{2} z$ so that $\|z\|=\varepsilon$. Then we have

$$
\begin{aligned}
|z(t)|= & \mu\left|\left(\mathcal{B}_{1} z\right)(t)\right|\left|\left(\mathcal{B}_{2} z\right)(t)\right|=\mu|h(t, z(t))| \\
& \times \mid-\int_{0}^{t} \frac{(t-\tau)^{p-1}}{\Gamma(p)} \Phi(\tau, z(\tau)) \mathrm{d} \tau+\lambda \int_{0}^{1} \Phi(\tau, z(\tau)) \mathrm{d} \tau \\
& +\int_{0}^{\eta} \frac{(\eta-\tau)^{p-1}}{\Gamma(p)} \Phi(\tau, z(\tau)) \mathrm{d} \tau \mid
\end{aligned}
$$




$$
\begin{aligned}
\leq & (|h(t, z(t))-h(t, 0)|+|h(t, 0)|) \\
& \times\left(\int_{0}^{t} \frac{(t-\tau)^{p-1}}{\Gamma(p)}|\Phi(\tau, z(\tau))| \mathrm{d} \tau+\lambda \int_{0}^{1}|\Phi(\tau, z(\tau))| \mathrm{d} \tau\right. \\
& \left.+\int_{0}^{\eta} \frac{(\eta-\tau)^{p-1}}{\Gamma(p)}|\Phi(\tau, z(\tau))| \mathrm{d} \tau\right) \\
\leq & \left(\kappa(t)|z(t)|+H^{*}\right) \Delta^{*} G^{*} \chi(\|z\|) \\
\leq & \left(\kappa^{*}\|z\|+H^{*}\right) \Delta^{*} G^{*} \chi(\|z\|)
\end{aligned}
$$

and so $\varepsilon \leq \frac{H^{*} \Delta^{*} G^{*} \chi(\|z\|)}{1-\kappa^{*} \Delta^{*} G^{*} \chi(\|z\|)}$ which is a contradiction to inequality (9). This means that the condition (b) of Lemma 1 is impossible. Thus, the condition (a) in Lemma 1 holds and the fractional hybrid problem (1)-(2) has a solution.

Now, we are going to state and prove our main result for the fractional hybrid inclusion model for the thermostat which is stated by (3)-(4).

Definition 6 We say that an absolutely continuous function $z:[0,1] \rightarrow \mathbb{R}$ is a solution for the fractional hybrid inclusion problem (3)-(4) whenever there is an integrable function $v \in \mathcal{L}^{1}([0,1], \mathbb{R})$ with $v(t) \in \mathcal{G}(t, z(t))$ for almost all $t \in[0,1]$,

$$
\left.\mathcal{D}\left(\frac{z(t)}{h(t, z(t))}\right)\right|_{t=0}=0,\left.\quad \lambda^{c} \mathcal{D}_{0}^{p-1}\left(\frac{z(t)}{h(t, z(t))}\right)\right|_{t=1}+\left.\left(\frac{z(t)}{h(t, z(t))}\right)\right|_{t=\eta}=0
$$

and

$$
z(t)=h(t, z(t))\left[-\int_{0}^{t} \frac{(t-\tau)^{p-1}}{\Gamma(p)} v(\tau) \mathrm{d} \tau+\int_{0}^{\eta} \frac{(\eta-\tau)^{p-1}}{\Gamma(p)} v(\tau) \mathrm{d} \tau+\lambda \int_{0}^{1} v(\tau) \mathrm{d} \tau\right]
$$

for all $t \in[0,1]$.

\section{Theorem 7 Suppose that}

$(\mathcal{A} 4)$ there exists a bounded mapping $\kappa:[0,1] \rightarrow \mathbb{R}^{+}$such that, for every $z_{1}, z_{2} \in \mathbb{R}$ and $t \in[0,1]$, we have $\left|h\left(t, z_{1}(t)\right)-h\left(t, z_{2}(t)\right)\right| \leq \kappa(t)\left|z_{1}(t)-z_{2}(t)\right| ;$

$(\mathcal{A} 5)$ the set-valued map $\mathcal{G}:[0,1] \times \mathbb{R} \rightarrow \mathcal{P}_{c p, c v}(\mathbb{R})$ has the $\mathcal{L}^{1}$-Carathéodory property;

(A6) there exists a positive mapping $\sigma \in \mathcal{L}^{1}\left([0,1], \mathbb{R}^{+}\right)$such that

$$
\|\mathcal{G}(t, z)\|=\sup \{|v|: v \in \mathcal{G}(t, z(t))\} \leq \sigma(t)
$$

for all $z \in \mathbb{R}$, almost all $t \in[0,1]$ and $\|\sigma\|_{\mathcal{L}^{1}}=\int_{0}^{1}|\sigma(\tau)| \mathrm{d} \tau$;

(A7) there exists a positive number $\tilde{\varepsilon} \in \mathbb{R}$ such that

$$
\tilde{\varepsilon}>\frac{H^{*} \Delta^{*}\|\sigma\|_{\mathcal{L}^{1}}}{1-\kappa^{*} \Delta^{*}\|\sigma\|_{\mathcal{L}^{1}}}
$$

where $H^{*}=\sup _{t \in[0,1]}|h(t, 0)|$ and $\kappa^{*}=\sup _{t \in[0,1]}|\kappa(t)|$.

Then the fractional hybrid inclusion problem (3)-(4) has at least one solution whenever $\kappa^{*} \Delta^{*}\|\sigma\|_{\mathcal{L}^{1}}<\frac{1}{2}$. 
Proof Let $t \in[0,1]$. Consider the operator $\mathcal{K}: \mathcal{M} \rightarrow \mathcal{P}(\mathcal{M})$ defined by

$$
\mathcal{K}(z)=\left\{\begin{array}{l}
w \in \mathcal{M}: \\
w(t)=\left\{\begin{array}{c}
h(t, z(t))\left(-\int_{0}^{t} \frac{(t-\tau)^{p-1}}{\Gamma(p)} v(\tau) \mathrm{d} \tau\right. \\
+\int_{0}^{\eta} \frac{(\eta-\tau)^{p-1}}{\Gamma(p)} v(\tau) \mathrm{d} \tau \\
\left.+\lambda \int_{0}^{1} v(\tau) \mathrm{d} \tau\right), \quad v \in(S E L)_{\mathcal{G}, z}
\end{array}\right.
\end{array}\right.
$$

Note that the fixed point of $\mathcal{K}$ is a solution for the fractional hybrid inclusion problem (3)-(4). Define a single-valued mapping $\mathcal{B}_{1}: \mathcal{M} \rightarrow \mathcal{M}$ by $\left(\mathcal{B}_{1} z\right)(t)=h(t, z(t))$ and the setvalued map $\mathcal{B}_{2}: \mathcal{M} \rightarrow \mathcal{P}(\mathcal{M})$ by

$$
\left(\mathcal{B}_{2} z\right)(t)=\left\{\begin{array}{l}
\psi \in \mathcal{M}: \\
\psi(t)=\left\{\begin{array}{c}
-\int_{0}^{t} \frac{(t-\tau)^{p-1}}{\Gamma(p)} v(\tau) \mathrm{d} \tau+\int_{0}^{\eta} \frac{(\eta-\tau)^{p-1}}{\Gamma(p)} v(\tau) \mathrm{d} \tau \\
+\lambda \int_{0}^{1} v(\tau) \mathrm{d} \tau, \quad v \in(S E L)_{\mathcal{G}, z}
\end{array}\right.
\end{array}\right.
$$

for $t \in[0,1]$. Note that $\mathcal{K}(z)=\mathcal{B}_{1} z \mathcal{B}_{2} z$. We prove that $\mathcal{B}_{1}$ and $\mathcal{B}_{2}$ satisfy the assumptions of Lemma 3. First by using the assumption $(\mathcal{A} 4)$ and similar proof in Theorem 5 , one can show that $\mathcal{B}_{1}$ is Lipschitz on $\mathcal{M}$. Now, we prove that the set-valued map $\mathcal{B}_{2}$ has convex values. Let $z_{1}, z_{2} \in \mathcal{B}_{2} z$. Then choose $v_{1}, v_{2} \in(S E L)_{\mathcal{G}, z}$ such that

$$
\begin{aligned}
z_{j}(t)= & -\int_{0}^{t} \frac{(t-\tau)^{p-1}}{\Gamma(p)} v_{j}(\tau) \mathrm{d} \tau+\lambda \int_{0}^{1} v_{j}(\tau) \mathrm{d} \tau \\
& +\int_{0}^{\eta} \frac{(\eta-\tau)^{p-1}}{\Gamma(p)} v_{j}(\tau) \mathrm{d} \tau \quad(j=1,2)
\end{aligned}
$$

for almost all $t \in[0,1]$. For each constant $\gamma \in(0,1)$, we have

$$
\begin{aligned}
\gamma z_{1}(t)+(1-\gamma) z_{2}(t)= & -\int_{0}^{t} \frac{(t-\tau)^{p-1}}{\Gamma(p)}\left[\gamma v_{1}(\tau)+(1-\gamma) v_{2}(\tau)\right] \mathrm{d} \tau \\
& +\lambda \int_{0}^{1}\left[\gamma v_{1}(\tau)+(1-\gamma) v_{2}(\tau)\right] \mathrm{d} \tau \\
& +\int_{0}^{\eta} \frac{(\eta-\tau)^{p-1}}{\Gamma(p)}\left[\gamma v_{1}(\tau)+(1-\gamma) v_{2}(\tau)\right] \mathrm{d} \tau
\end{aligned}
$$

for almost all $t \in[0,1]$. Since $\mathcal{G}$ is convex-valued, $(S E L)_{\mathcal{G}, z}$ has convex values and so $\gamma v_{1}(t)+$ $(1-\gamma) \nu_{2}(t) \in(S E L)_{\mathcal{G}, z}$ for all $t \in[0,1]$. Thus, $\mathcal{B}_{2} z$ is a convex set for all $z \in \mathcal{M}$.

To establish the complete continuity of the operator $\mathcal{B}_{2}$, we must prove that $\mathcal{B}_{2}(\mathcal{M})$ is an equi-continuous and uniformly bounded set. To do this, we prove that $\mathcal{B}_{2}$ maps all bounded sets into bounded subsets of the space $\mathcal{M}$. For a positive number $\varepsilon^{*} \in \mathbb{R}$, consider a bounded ball $V_{\varepsilon^{*}}=\left\{z \in \mathcal{M}:\|z\|_{\mathcal{M}} \leq \varepsilon^{*}\right\}$. For every $z \in V_{\varepsilon^{*}}$ and $\psi \in \mathcal{B}_{2} z$, there is a function $v \in(S E L)_{\mathcal{G}, z}$ such that

$$
\begin{aligned}
\psi(t)= & -\int_{0}^{t} \frac{(t-\tau)^{p-1}}{\Gamma(p)} v(\tau) \mathrm{d} \tau+\lambda \int_{0}^{1} v(\tau) \mathrm{d} \tau \\
& +\int_{0}^{\eta} \frac{(\eta-\tau)^{p-1}}{\Gamma(p)} v(\tau) \mathrm{d} \tau
\end{aligned}
$$


for all $t \in[0,1]$. Then we have

$$
\begin{aligned}
|\psi(t)| \leq & \int_{0}^{t} \frac{(t-\tau)^{p-1}}{\Gamma(p)}|v(\tau)| \mathrm{d} \tau+\lambda \int_{0}^{1}|v(\tau)| \mathrm{d} \tau \\
& +\int_{0}^{\eta} \frac{(\eta-\tau)^{p-1}}{\Gamma(p)}|v(\tau)| \mathrm{d} \tau \\
\leq & \int_{0}^{t} \frac{(t-\tau)^{p-1}}{\Gamma(p)} \sigma(\tau) \mathrm{d} \tau+\lambda \int_{0}^{1} \sigma(\tau) \mathrm{d} \tau \\
& +\int_{0}^{\eta} \frac{(\eta-\tau)^{p-1}}{\Gamma(p)} \sigma(\tau) \mathrm{d} \tau \\
\leq & {\left[\frac{1}{\Gamma(p+1)}+\frac{\eta^{p}}{\Gamma(p+1)}+\lambda\right]\|\sigma\|_{\mathcal{L}^{1}} } \\
= & \Delta^{*}\|\sigma\|_{\mathcal{L}^{1}}
\end{aligned}
$$

where $\Delta^{*}$ is given in (10). Thus, $\|\psi\| \leq \Delta^{*}\|\sigma\|_{\mathcal{L}^{1}}$ and this implies that $\mathcal{B}_{2}(\mathcal{M})$ is a uniformly bounded set. Now, we show that the operator $\mathcal{B}_{2}$ maps bounded sets onto equicontinuous sets. Let $z \in V_{\varepsilon^{*}}$ and $\psi \in \mathcal{B}_{2} z$. Choose $v \in(S E L)_{\mathcal{G}, z}$ such that

$$
\begin{aligned}
\psi(t)= & -\int_{0}^{t} \frac{(t-\tau)^{p-1}}{\Gamma(p)} v(\tau) \mathrm{d} \tau+\lambda \int_{0}^{1} \nu(\tau) \mathrm{d} \tau \\
& +\int_{0}^{\eta} \frac{(\eta-\tau)^{p-1}}{\Gamma(p)} v(\tau) \mathrm{d} \tau
\end{aligned}
$$

for all $t \in[0,1]$. For each $t_{1}, t_{2} \in[0,1]$ with $t_{1}<t_{2}$, we have

$$
\begin{aligned}
\left|\psi\left(t_{2}\right)-\psi\left(t_{1}\right)\right| \leq & \mid \int_{0}^{t_{2}} \frac{\left(t_{2}-\tau\right)^{p-1}}{\Gamma(p)} v(\tau) \mathrm{d} \tau \\
& -\int_{0}^{t_{1}} \frac{\left(t_{1}-\tau\right)^{p-1}}{\Gamma(p)} v(\tau) \mathrm{d} \tau \mid \\
\leq & \int_{0}^{t_{1}}\left(\frac{\left[\left(t_{2}-\tau\right)^{p-1}-\left(t_{1}-\tau\right)^{p-1}\right]}{\Gamma(p)}\right) \sigma(\tau) \mathrm{d} \tau \\
& +\int_{t_{1}}^{t_{2}} \frac{\left(t_{2}-\tau\right)^{p-1}}{\Gamma(p)} \sigma(\tau) \mathrm{d} \tau .
\end{aligned}
$$

It is obvious that the right-hand side of the above inequalities towards to zero independently of $z \in V_{\varepsilon^{*}}$ as $t_{2} \rightarrow t_{1}$. Hence by using the Arzela-Ascoli theorem, the operator $\mathcal{B}_{2}: C([0,1], \mathbb{R}) \rightarrow \mathcal{P}(C([0,1], \mathbb{R}))$ has the complete continuity property.

Now, we show that $\mathcal{B}_{2}$ has a closed graph and this implies that $\mathcal{B}_{2}$ is upper semicontinuous because $\mathcal{B}_{2}$ is completely continuous. For this goal, assume that $z_{n} \in V_{\varepsilon^{*}}$ and $\psi_{n} \in \mathcal{B}_{2} z_{n}$ are such that $z_{n} \rightarrow z^{*}$ and $\psi_{n} \rightarrow \psi^{*}$. We claim that $\psi^{*} \in \mathcal{B}_{2} z^{*}$. For each $n \geq 1$ and $\psi_{n} \in \mathcal{B}_{2} z_{n}$, choose $v_{n} \in(S E L)_{\mathcal{G}, z_{n}}$ such that

$$
\begin{aligned}
\psi_{n}(t)= & -\int_{0}^{t} \frac{(t-\tau)^{p-1}}{\Gamma(p)} v_{n}(\tau) \mathrm{d} \tau+\lambda \int_{0}^{1} v_{n}(\tau) \mathrm{d} \tau \\
& +\int_{0}^{\eta} \frac{(\eta-\tau)^{p-1}}{\Gamma(p)} v_{n}(\tau) \mathrm{d} \tau
\end{aligned}
$$


for all $t \in[0,1]$. It is sufficient to show that there exists a function $v^{*} \in(S E L)_{\mathcal{G}, z^{*}}$ such that

$$
\begin{aligned}
\psi^{*}(t)= & -\int_{0}^{t} \frac{(t-\tau)^{p-1}}{\Gamma(p)} \nu^{*}(\tau) \mathrm{d} \tau+\lambda \int_{0}^{1} \nu^{*}(\tau) \mathrm{d} \tau \\
& +\int_{0}^{\eta} \frac{(\eta-\tau)^{p-1}}{\Gamma(p)} \nu^{*}(\tau) \mathrm{d} \tau
\end{aligned}
$$

for all $t \in[0,1]$. Suppose that the continuous linear operator

$$
\Upsilon: \mathcal{L}^{1}([0,1], \mathbb{R}) \rightarrow \mathcal{M}=C([0,1], \mathbb{R})
$$

is defined by

$$
\begin{aligned}
\Upsilon(v)(t)=z(t)= & -\int_{0}^{t} \frac{(t-\tau)^{p-1}}{\Gamma(p)} v(\tau) \mathrm{d} \tau+\lambda \int_{0}^{1} v(\tau) \mathrm{d} \tau \\
& +\int_{0}^{\eta} \frac{(\eta-\tau)^{p-1}}{\Gamma(p)} v(\tau) \mathrm{d} \tau
\end{aligned}
$$

for all $t \in[0,1]$. Then we have

$$
\begin{aligned}
\left\|\psi_{n}(t)-\psi^{*}(t)\right\|= & \|-\int_{0}^{t} \frac{(t-\tau)^{p-1}}{\Gamma(p)}\left(v_{n}(\tau)-v^{*}(\tau)\right) \mathrm{d} \tau \\
& +\lambda \int_{0}^{1}\left(v_{n}(\tau)-v^{*}(\tau)\right) \mathrm{d} \tau \\
& +\int_{0}^{\eta} \frac{(\eta-\tau)^{p-1}}{\Gamma(p)}\left(v_{n}(\tau)-v^{*}(\tau)\right) \mathrm{d} \tau \| \rightarrow 0
\end{aligned}
$$

as $n \rightarrow \infty$. Hence by using Lemma 2, we conclude that the operator $\Upsilon \circ(S E L)_{\mathcal{G}}$ has a closed graph. Since $\psi_{n} \in \Upsilon\left((S E L)_{\mathcal{G}, z_{n}}\right)$ and $z_{n} \rightarrow z^{*}$, there exists $v^{*} \in(S E L)_{\mathcal{G}, z^{*}}$ such that

$$
\begin{aligned}
\psi^{*}(t)= & -\int_{0}^{t} \frac{(t-\tau)^{p-1}}{\Gamma(p)} \nu^{*}(\tau) \mathrm{d} \tau+\lambda \int_{0}^{1} \nu^{*}(\tau) \mathrm{d} \tau \\
& +\int_{0}^{\eta} \frac{(\eta-\tau)^{p-1}}{\Gamma(p)} \nu^{*}(\tau) \mathrm{d} \tau
\end{aligned}
$$

for all $t \in[0,1]$. Thus, $\psi^{*} \in \mathcal{B}_{2} z^{*}$ and so $\mathcal{B}_{2}$ has a closed graph. Hence, $\mathcal{B}_{2}$ is upper semicontinuous. Moreover, by the hypothesis, the operator $\mathcal{B}_{2}$ has compact values. Consequently, $\mathcal{B}_{2}$ is a compact and upper semi-continuous operator. Now by using the assumption $(\mathcal{A} 6)$, we get

$$
\begin{aligned}
M_{0}^{*} & =\left\|\mathcal{B}_{2}(\mathcal{M})\right\|=\sup \left\{\left|\mathcal{B}_{2} z\right|: z \in \mathcal{M}\right\} \\
& =\left[\frac{1}{\Gamma(p+1)}+\frac{\eta^{p}}{\Gamma(p+1)}+\lambda\right]\|\sigma\|_{\mathcal{L}^{1}} \\
& =\Delta^{*}\|\sigma\|_{\mathcal{L}^{1}} .
\end{aligned}
$$

By putting $l^{*}=\kappa^{*}$, we obtain $M_{0}^{*} l^{*}<\frac{1}{2}$. Therefore, the assumptions of Lemma 3 hold for $\mathcal{B}_{1}$ and $\mathcal{B}_{2}$ and so one of the conditions (a) or (b) holds. 
We claim that the condition (b) is impossible. By using Lemma 3 and the assumption $(\mathcal{A} 7)$, assume that $z$ is an arbitrary element of $\Sigma^{*}$ with $\|z\|=\tilde{\varepsilon}$. Then $\mu z(t) \in \mathcal{B}_{1} z(t) \mathcal{B}_{2} z(t)$ for all $\mu>1$. Select the related function $v \in(S E L)_{\mathcal{G}, z}$. Then, for each $\mu>1$, we obtain

$$
\begin{aligned}
z(t)= & \frac{1}{\mu} h(t, z(t))\left[-\int_{0}^{t} \frac{(t-\tau)^{p-1}}{\Gamma(p)} v(\tau) \mathrm{d} \tau+\lambda \int_{0}^{1} v(\tau) \mathrm{d} \tau\right. \\
& \left.+\int_{0}^{\eta} \frac{(\eta-\tau)^{p-1}}{\Gamma(p)} v(\tau) \mathrm{d} \tau\right]
\end{aligned}
$$

for all $t \in[0,1]$. Thus, we get

$$
\begin{aligned}
|z(t)|= & \frac{1}{\mu}|h(t, z(t))|\left[\int_{0}^{t} \frac{(t-\tau)^{p-1}}{\Gamma(p)}|v(\tau)| \mathrm{d} \tau\right. \\
& \left.+\lambda \int_{0}^{1}|v(\tau)| \mathrm{d} \tau+\int_{0}^{\eta} \frac{(\eta-\tau)^{p-1}}{\Gamma(p)}|v(\tau)| \mathrm{d} \tau\right] \\
\leq & {[|h(t, z(t))-h(t, 0)|+|h(t, 0)|]\left[\int_{0}^{t} \frac{(t-\tau)^{p-1}}{\Gamma(p)}|v(\tau)| \mathrm{d} \tau\right.} \\
& +\lambda \int_{0}^{1}|v(\tau)| \mathrm{d} \tau \\
& \left.+\int_{0}^{\eta} \frac{(\eta-\tau)^{p-1}}{\Gamma(p)}|v(\tau)| \mathrm{d} \tau\right] \\
\leq & {\left[\kappa^{*}\|z\|+H^{*}\right]\left[\int_{0}^{t} \frac{(t-\tau)^{p-1}}{\Gamma(p)} \sigma(\tau) \mathrm{d} \tau\right.} \\
& \left.+\lambda \int_{0}^{1} \sigma(\tau) \mathrm{d} \tau+\int_{0}^{\eta} \frac{(\eta-\tau)^{p-1}}{\Gamma(p)} \sigma(\tau) \mathrm{d} \tau\right] \\
\leq & {\left[\kappa^{*} \tilde{\varepsilon}+H^{*}\right] \Delta^{*}\|\sigma\|_{\mathcal{L}^{1}} }
\end{aligned}
$$

for all $t \in[0,1]$. Hence,

$$
\tilde{\varepsilon} \leq \frac{H^{*} \Delta^{*}\|\sigma\|_{\mathcal{L}^{1}}}{1-\kappa^{*} \Delta^{*}\|\sigma\|_{\mathcal{L}^{1}}}
$$

By using the condition (11), we observe that the condition (b) of Lemma 3 is impossible. Thus, the operator inclusion $z \in \mathcal{B}_{1} z \mathcal{B}_{2} z$ has a solution and so the fractional hybrid inclusion problem (3)-(4) has at least one solution.

Now, we provide two examples to illustrate our main results.

Example 1 Consider the fractional hybrid differential equation of the thermostat model

$$
{ }^{c} \mathcal{D}_{0}^{\frac{3}{2}}\left(\frac{z(t)}{\frac{t|z(t)|^{2}}{6+|z(t)|^{2}}+5}\right)+\frac{t \sin ^{2}\left(\frac{\pi}{2}(t)\right) \sin (z(t))}{1000}=0 \quad(t \in[0,1])
$$


with the three-point hybrid conditions

$$
\begin{aligned}
& \left.\mathcal{D}\left(\frac{z(t)}{\frac{t|z(t)|^{2}}{6+|z(t)|^{2}}+5}\right)\right|_{t=0}=0, \\
& \left.\frac{1}{10} \mathcal{D}_{0}^{\frac{1}{2}}\left(\frac{z(t)}{\frac{t|z(t)|^{2}}{6+|z(t)|^{2}}+5}\right)\right|_{t=1}+\left.\left(\frac{z(t)}{\frac{t|z(t)|^{2}}{6+|z(t)|^{2}}+5}\right)\right|_{t=\frac{1}{4}}=0 .
\end{aligned}
$$

Put $p=\frac{3}{2}, p-1=\frac{1}{2}, \eta=\frac{1}{4}$ and $\lambda=\frac{1}{10}$. Consider the continuous maps $h:[0,1] \times \mathbb{R} \rightarrow \mathbb{R} \backslash\{0\}$ and $\Phi:[0,1] \times \mathbb{R} \rightarrow \mathbb{R}^{+}$defined by

$$
h(t, z(t))=\frac{t|z(t)|^{2}}{6+|z(t)|_{2}}+5
$$

and

$$
\Phi(t, z(t))=\frac{t \sin ^{2}\left(\frac{\pi}{2}(t)\right) \sin (z(t))}{1000} .
$$

If $\kappa(t)=t$, then $\kappa^{*}=\sup _{t \in[0,1]}|\kappa(t)|=1$. Put $g(t)=\frac{1}{1000}$ and $\chi(\|z\|)=1$. Then $\Delta^{*} \simeq 0.85238$. Choose $\varepsilon>0.00426$. Then $\kappa^{*} \Delta^{*} G^{*} \chi(\|z\|) \simeq 0.00085<1$. Now by using Theorem 5 , the fractional hybrid equation (12) with the three-point hybrid conditions (13) has at least one solution.

Example 2 Consider the fractional hybrid inclusion problem

$$
{ }^{c} \mathcal{D}_{0}^{\frac{8}{5}}\left(\frac{z(t)}{t \cos \frac{z(t)}{100}+6}\right) \in\left[\frac{|z(t)|}{4(|z(t)|+1)}+\frac{1}{2}, \frac{|\sin z(t)|^{4}}{5\left(1+|\sin z(t)|^{4}\right)}+\frac{4}{5}\right] \quad(t \in[0,1]),
$$

with the three-point hybrid conditions

$$
\begin{aligned}
& \left.\mathcal{D}\left(\frac{z(t)}{t \cos \frac{z(t)}{100}+6}\right)\right|_{t=0}=0, \\
& \left.\frac{7}{4} \mathcal{D}_{0}^{\frac{3}{5}}\left(\frac{z(t)}{t \cos \frac{z(t)}{100}+6}\right)\right|_{t=1}+\left.\left(\frac{z(t)}{t \cos \frac{z(t)}{100}+6}\right)\right|_{t=0.89}=0 .
\end{aligned}
$$

Put $p=\frac{8}{5}, p-1=\frac{3}{5}, \eta=0.89$ and $\lambda=\frac{7}{4}$. Define the continuous map $h:[0,1] \times \mathbb{R} \rightarrow \mathbb{R} \backslash\{0\}$ by $h(t, z(t))=t \cos \frac{z(t)}{100}+6$ and the set-valued $\operatorname{map} \mathcal{G}:[0,1] \times \mathbb{R} \rightarrow \mathcal{P}(\mathbb{R})$ by

$$
\mathcal{G}(t, z(t))=\left[\frac{|z(t)|}{4(|z(t)|+1)}+\frac{1}{2}, \frac{|\sin z(t)|^{4}}{5\left(1+|\sin z(t)|^{4}\right)}+\frac{4}{5}\right] .
$$

If $\kappa(t)=\frac{t}{100}$, then $\kappa^{*}=\sup _{t \in[0,1]}|\kappa(t)|=\frac{1}{100}$. Since

$$
|\psi| \leq \max \left(\frac{|z(t)|}{4(|z(t)|+1)}+\frac{1}{2}, \frac{|\sin z(t)|^{4}}{5\left(1+|\sin z(t)|^{4}\right)}+\frac{4}{5}\right) \leq 1 \quad(z \in \mathbb{R})
$$

for all $\psi \in \mathcal{G}(t, z(t))$, we get $\|\mathcal{G}(t, z(t))\|=\sup \{|v|: v \in \mathcal{G}(t, z(t))\} \leq 1$. Put $\sigma(t)=1$ for all $t \in[0,1]$ and so $\|\sigma\|_{\mathcal{L}^{1}}=1$. By using the above relations, we get $\Delta^{*} \simeq 3.0298$. Hence, we 
can find $\tilde{\varepsilon}>0$ with $\tilde{\varepsilon}>21.8712$. Since $\kappa^{*} \Delta^{*}\|\sigma\|_{\mathcal{L}^{1}} \simeq 0.030298<\frac{1}{2}$, by using Theorem 7, the fractional hybrid inclusion problem (14)-(15) has at least one solution.

\section{Conclusion}

It is important that we increase our ability for studying complicated fractional integrodifferential equations. One of the methods is extending well-known models to different complicated versions. In this work, we provide an extension for the well-known fractional thermostat model to its hybrid equation and inclusion versions. We also consider boundary value conditions of this problem in the form of the hybrid conditions. Finally, we provide two examples to illustrate our main results.

\section{Acknowledgements}

The second and third authors were supported by Azarbaijan Shahid Madani University. The authors express their gratitude to the unknown referees for their helpful suggestions, which improved the final version of this paper.

\section{Funding}

Not applicable.

Availability of data and materials

Data sharing not applicable to this article as no datasets were generated or analyzed during the current study.

\section{Ethics approval and consent to participate}

Not applicable.

Competing interests

The authors declare that they have no competing interests.

Consent for publication

Not applicable.

\section{Authors' contributions}

The authors declare that the study was realized in collaboration with equal responsibility. All authors read and approved the final manuscript.

\section{Author details}

'Department of Mathematics, Cankaya University, Ankara, Turkey. ${ }^{2}$ Institute of Space Sciences, Magurele, Bucharest, Romania. ${ }^{3}$ Department of Mathematics, Azarbaijan Shahid Madani University, Tabriz, Iran. ${ }^{4}$ Department of Medical Research, China Medical University Hospital, China Medical University, Taichung, Taiwan.

\section{Publisher's Note}

Springer Nature remains neutral with regard to jurisdictional claims in published maps and institutional affiliations.

Received: 6 January 2020 Accepted: 16 March 2020 Published online: 24 March 2020

\section{References}

1. Baleanu, D., Etemad, S., Pourrazi, S., Rezapour, S.: On the new fractional hybrid boundary value problems with three-point integral hybrid conditions. Adv. Differ. Equ. 2019, 473 (2019)

2. Baleanu, D., Khan, H., Jafari, H., Khan, R.A., Alipour, M.: On existence results for solutions of a coupled system of hybrid boundary value problems with hybrid conditions. Adv. Differ. Equ. 2015, 318 (2015)

3. Hilal, K., Kajouni, A.: Boundary value problems for hybrid differential equations with fractional order. Adv. Differ. Equ. 2015, $183(2015)$

4. Zhao, Y., Sun, S., Han, Z., Li, Q.: Theory of fractional hybrid differential equations. Comput. Math. Appl. 62(3), 1312-1324 (2011). https://doi.org/10.1016/j.camwa.2011.03.041

5. Baleanu, D., Hedayati, V., Rezapour, S., Al Qurashi, M.M.: On two fractional differential inclusions. SpringerPlus 5(1), 882 (2016). https://doi.org/10.1186/s40064-016-2564-z

6. Dhage, B.C., Lakshmikantham, V:: Basic results on hybrid differential equation. Nonlinear Anal. Hybrid Syst. 4, 414-424 (2010)

7. Infante, G., Webb, J.: Loss of positivity in a nonlinear scalar heat equation. Nonlinear Differ. Equ. Appl. 13, 249-261 (2006)

8. Nieto, J.J., Pimentel, J.: Positive solutions of a fractional thermostat model. Bound. Value Probl. 2013, 5 (2013)

9. Kontes, G.D., Giannakis, G.I., Horn, P., Steiger, S., Rovas, D.V.: Using thermostats for indoor climate control in office buildings: the effect on thermal comfort. Energy J. 33(4), 61-96 (2012)

10. Liang, Y., Levine, D.I., Shen, Z.J.: Thermostats for the smart grid: models, benchmarks and insights. Energies 10,1368 (2017). https://doi.org/10.3390/en10091368 
11. Urban, B., Gomez, C.: A case for thermostat user models. In: Proceedings of 13th Conf. Inter. Building Perform. Simulation Assoc, France, August 26-28, pp. 1483-1490 (2013)

12. Podlubny, I.: Fractional Differential Equations. Academic Press, San Diego (1999)

13. Samko, S.G., Kilbas, A.A., Marichev, O.I.: Fractional Integrals and Derivatives: Theory and Applications. Gordon and Breach Science Publishers, Switzerland (1993)

14. Miller, K.S., Ross, B.: An Introduction to Fractional Calculus and Fractional Differential Equations. Wiley, New York (1993)

15. Deimling, K.: Multi-Valued Differential Equations. Walter de Gruyter, Berlin (1992)

16. Aubin, J., Ceuina, A.: Differential Inclusions: Set-Valued Maps and Viability Theory. Springer, Berlin (1984). https://doi.org/10.1007/978-3-642-69512-4

17. Dhage, B.C.: Nonlinear functional boundary value problems involving Carathéodory. Kyungpook Math. J. 46, 427-441 (2006)

18. Lasota, A., Opial, Z:: An application of the Kakutani-Ky Fan theorem in the theory of ordinary differential equations. Bull. Acad. Pol. Sci., Sér. Sci. Math. Astron. Phys. 13, 781-786 (1965)

19. Dhage, B.C.: Existence results for neutral functional differential inclusions in Banach algebras. Nonlinear Anal. 64 1290-1306 (2006)

\section{Submit your manuscript to a SpringerOpen ${ }^{0}$ journal and benefit from:}

- Convenient online submission

- Rigorous peer review

- Open access: articles freely available online

- High visibility within the field

- Retaining the copyright to your article 